\title{
Antitumor activity of chemoendocrine therapy in premenopausal and postmenopausal models with human breast cancer xenografts
}

\author{
MOTOYUKI KATAOKA ${ }^{1}$, YURI YAMAGUCHI ${ }^{2}$, YOICHIRO MORIYA ${ }^{1}$, NORIAKI SAWADA ${ }^{1}$, HIDEYUKI YASUNO ${ }^{1}$, \\ KUMIKO KONDOH ${ }^{1}$, DEAN B. EVANS ${ }^{3}$, KAZUSHIGE MORI ${ }^{1}$ and SHIN-ICHI HAYASHI ${ }^{4}$ \\ ${ }^{1}$ Product Research Department, Kamakura Research Laboratories, Chugai Pharmaceutical Co., Ltd., Kamakura 247-8530; \\ ${ }^{2}$ Research Institute for Clinical Oncology, Saitama Cancer Center, Saitama 362-0806, Japan; ${ }^{3}$ Oncology Research, Novartis \\ Institutes for BioMedical Research, Basel, Switzerland; ${ }^{4}$ Center for Regulatory Epigenome and Diseases, Molecular and \\ Functional Dynamics, Graduate School of Medicine, Tohoku University, Sendai 980-8575, Japan
}

Received August 26, 2011; Accepted September 22, 2011

DOI: 10.3892/or.2011.1541

\begin{abstract}
We examined the efficacy of chemoendocrine therapy using capecitabine as a chemotherapeutic agent in premenopausal and postmenopausal models with estrogen receptor (ER)-positive human breast cancer xenografts. Tamoxifen and letrozole were used as endocrine therapeutic agents for premenopausal and postmenopausal models, respectively. The antitumor activity of capecitabine in combination was significantly superior to either monotherapy treatment in both premenopausal $(\mathrm{p}<0.01)$ and postmenopausal $(\mathrm{p}<0.05)$ models. No increase in toxicity in terms of body weight loss was observed during treatment in either of the xenograft models. In the premenopausal model, the level of thymidine phosphorylase (TP), a key enzyme generating 5-FU from capecitabine, was upregulated $(\mathrm{p}<0.05)$ in tumors by tamoxifen but not by letrozole treatment in the postmenopausal model. The combination of 5'-deoxy-5-fluorouridine (5'-DFUR; an intermediate of capecitabine) with 4-hydroxytamoxifen (4-OHT; an active form of tamoxifen) or letrozole was also evaluated in vitro by using estrogen-responsive element (ERE) reporter gene assays aimed to model premenopausal and postmenopausal breast cancer. Both combinations decreased the number of estrogenresponding cells in a concentration-dependent manner and further analysis by isobolograms revealed a synergistic effect of the combination of 5'-DFUR with 4-OHT, and at least an additive effect of the combination of 5'-DFUR with letrozole. These results suggest that chemoendocrine therapy using capecitabine may be a useful treatment modality for patients with hormone-receptor-positive breast cancer, regardless of the menopausal status and should be explored in clinical trials.
\end{abstract}

Correspondence to: Dr Yoichiro Moriya, Product Research Department, Kamakura Research Laboratories, Chugai Pharmaceutical Co. Ltd., 200 Kajiwara, Kamakura 247-8530, Japan

E-mail: moriyayui@chugai-pharm.co.jp

Key words: capecitabine, tamoxifen, letrozole, chemoendocrine therapy, breast cancer

\section{Introduction}

Two decades ago, in vitro antagonism in cytotoxicity was reported between chemotherapeutic agents and antiestrogens in breast cancer cells (1). This observation translated to the clinic as results with chemoendocrine therapies in patients have been disappointing $(2,3)$. However, not all reports of addition of chemotherapies, such as CMF [a chemotherapy regimen consisting of cyclophosphamide, methotrexate and 5-fluorouracil (5-FU)], to endocrine therapies showed negative results. For example, clinical results from NSABP B-20 trial (4) and the International Breast Cancer Study Group (IBCSG) trial VII (5) indicated that the addition of CMF to tamoxifen yielded superior five-year disease-free survival rate. Therefore, it is likely that some chemotherapeutic agents may not be antagonistic to antiestrogens.

Capecitabine is an oral 5-FU derivative widely used for breast cancers which generates the active substance 5-FU in tumors by a three-step cascade of enzymes located in the liver and tumors. The final step is the conversion of 5'-DFUR, an intermediate metabolite, to 5-FU by TP, which is highly expressed in tumors. Therefore, a higher level of 5-FU would be expected in tumor tissues which have higher expression levels of TP, when treated with capecitabine. Indeed, it has been reported that the antitumor activity of capecitabine did correlate with tumor levels of TP activity in xenograft models, whereas that of 5-FU did not (6).

Estrogen is known to play a crucial role in estrogen receptor (ER)-positive breast cancer development and growth through binding to ER. It is reported that $71 \%$ of invasive breast cancer is ER-positive (7). Antiestrogen therapies are an indispensable treatment modality for almost all ER-positive breast cancer patients. Tamoxifen, an estrogen antagonist, was widely used for the treatment of ER-positive breast cancer patients with premenopausal, as well as postmenopausal, status. It was reported in a P-1 study that tamoxifen reduced the cumulative rate of invasive breast cancer (8), and that it reduced the incidence of contralateral breast cancer in premenopausal patients (9).

In postmenopausal women, the blood estrogen levels decrease because the ovaries cease to produce estrogen. However, estrogen level in breast cancer is still 10 times higher 
than that in normal breast tissue $(10,11)$ because estrogen is supplied through the conversion of androgen by aromatase, a key enzyme of estrogen synthesis. In addition, aromatase is known to be highly expressed in the adipose stromal cells adjacent to breast cancer cells (12). Thus, the locally produced estrogen promotes the growth of ER-positive tumor cells in postmenopausal breast cancer patients. Letrozole, an aromatase inhibitor, improved disease-free survival and time to distant recurrence compared to tamoxifen in postmenopausal ER-positive breast cancer patients (BIG 1-98 study) $(13,14)$.

In the present study, we examined the antitumor activity of capecitabine in combination with endocrine therapies in both premenopausal and postmenopausal breast cancer xenograft models. We also examined the effect of the combination capecitabine plus antiestrogens in in vitro ERE reporter gene assays $(15,16)$.

\section{Materials and methods}

Chemicals. Capecitabine and 5'-DFUR were synthesized at Hoffmann-La Roche (Basel, Switzerland). Tamoxifen citrate, 4-hydroxytamoxifen (4-OHT) and androstenedione were purchased from Sigma-Aldrich Co. (St. Louis, MO). Letrozole was obtained from Novartis Pharmaceutical Co., Ltd.

For in vivo study, capecitabine was dissolved in $40 \mathrm{mmol} / \mathrm{l}$ citrate buffer, $\mathrm{pH} 6.0$ containing $5 \%$ gum arabic. Tamoxifen was dissolved in the same buffer as capecitabine solution. Letrozole was dissolved in dimethyl sulfoxide, and further diluted with $0.5 \%$ aqueous solution of sodium carboxymethylcellulose containing $20 \%$ propylene glycol. For in vitro study, 5'-DFUR was dissolved in phosphate-buffered saline $(10 \mathrm{mmol} / \mathrm{l}) .4-\mathrm{OHT}$ was dissolved in ethanol $(1 \mathrm{mmol} / \mathrm{l})$, and further diluted with phosphate-buffered saline, $\mathrm{pH}$ 7.4. Letrozole was dissolved in the same manner as in the in vivo study.

Animals. Female 4-5-week-old BALB/c-nu/nu mice [CAnN. Cg-Foxn1(nu)/CrlCrlj nu/nu] were obtained from Charles River Laboratories Japan, Inc. (Yokohama, Japan). All mice were housed in a pathogen-free environment under controlled conditions (temperature $20-26^{\circ} \mathrm{C}$, humidity $40-70 \%$, light/dark cycle $12 \mathrm{~h} / 12 \mathrm{~h}$ ). Chlorinated water and irradiated food (CE-2; Clea Japan, Inc., Tokyo, Japan) were provided ad libitum. All mice were allowed to acclimatize and recover from shippingrelated stress for at least 1 week prior to the study. The health of the mice was monitored by daily observation. The protocol was reviewed by the Institutional Animal Care and Use Committee of Chugai Pharmaceutical Co., Ltd. and all mouse experiments were performed in accordance with the Guidelines for the Accommodation and Care of Laboratory Animals promulgated in Chugai Pharmaceutical Co., Ltd.

Cell lines and culture conditions. Estrogen receptor-positive MCF-7 human breast cancer cell line was kindly provided by Dr Y. Iino (Gunma University, Maebashi, Japan) and was maintained in Eagle's minimum essential medium (MEM; Sigma-Aldrich Co.) supplemented with $0.1 \mathrm{mmol} / 1 \mathrm{MEM}$ non-essential amino acids (Invitrogen Corp., Carlsbad, CA), $1 \mathrm{mmol} / 1$ sodium pyruvate (Invitrogen Corp.) and 10\% (v/v) heat-inactivated fetal bovine serum (FBS; Thermo Trace Ltd., Victoria, Australia).
Preparation of aromatase transfectant. The aromatase gene transfectant cell line MCF-7A25F3 was established according to the method of Zhou et al (17). Briefly, the first-strand cDNA synthesis was carried out with $5 \mu \mathrm{g}$ of total RNA from human placenta (Toyobo Co., Ltd., Osaka, Japan), 100 pmoles of oligo (dT) and 200 units of SuperScript II (Invitrogen Corp.). The target cDNA for aromatase gene was obtained by the polymerase chain reaction. The sequences of the primer were as follows: forward; 5'-GCT CTA GAG GAA CAC AAG ATG GTT TTG GAA-3' containing $X b a$ I restriction site for cloning, reverse; 5'-GCT CTA GAT GGG TAC TGA CCA GCC TTC T-3'. The PCR product was digested with $\mathrm{XbaI}$ and then was extracted from the gel. The cDNA fragment was inserted into the $X b a \mathrm{I}$ and $S m a \mathrm{I}$ site of the mammalian expression vector pCI-Neo (Promega Co., Ltd., WI). The plasmid DNA was purified using DNA miniprep column (Qiagen GmbH, Hilden, Germany) according to the manufacturer's instruction and then sequenced.

This sequence-confirmed construct was used for the transfection to generate the MCF-7 cell line expressing aromatase gene. To establish a stably transfected cell line, $1 \mu \mathrm{g}$ of plasmid DNA was incubated with $0.5 \mathrm{ml}$ of Lipofectamine (Clontech Laboratories Inc., CA) for $30 \mathrm{~min}$ and overlaid onto $1 \times 10^{6} \mathrm{MCF}-7$ cells for $1 \mathrm{~h}$ in serum-free medium. Cells were then selected in the presence of $350 \mu \mathrm{g} / \mathrm{ml}$ Geneticin (G-418; Calbiochem Co., Ltd., San Diego, CA). After selection for 2 weeks, the cells were screened for aromatase cDNA in genomic DNA by PCR. The ratio of copy numbers of aromatase cDNA in the parent MCF-7 cells and in the transfected cells were estimated. Primers for quantification were as follows: forward; 5'-GGA AAT GCT GAA CCC GAT AC-3', reverse; 5'-GAG AAA AAG GCC AGT GAG GA-3' for aromatase, and forward; 5'-GGT TGG CCA ATC TAC TCC CAG G-3', reverse; 5'-TGG TCT CCT TAA ACC TGT CTT G-3' for $\beta$-globin. The copy number of the aromatase gene of the transfected cells as a whole was 2-fold higher than that of the parent MCF-7 cells. The cloning of transfected cells was performed and clones revealing a high proliferation response to androstenedione were selected. Next, the clones were inoculated into ovariectomized BALB/c-nu/nu mice which had been implanted with a slow-release androstenedione pellet to determine the tumor growth in response to the hormone in vivo.

Measurement of aromatase activity. Aromatase activity was determined by using a tritiated water $\left({ }^{3} \mathrm{H}_{2} \mathrm{O}\right)$ method in which the ${ }^{3} \mathrm{H}_{2} \mathrm{O}$ released from $1 \beta-{ }^{3} \mathrm{H}$-androstenedione was measured. The measurement was conducted by Teikoku Hormone Medical Co., Ltd. (Kawasaki, Japan). Briefly, the collected MCF-7A25F3 cells were homogenized and centrifuged at $200 \mathrm{x} \mathrm{g}$ for $3 \mathrm{~min}$. The supernatant $(0.5 \mathrm{ml})$ was incubated with $1 \beta-{ }^{3} \mathrm{H}$-androstenedione and NADPH at $37^{\circ} \mathrm{C}$ for $60 \mathrm{~min}$. The incubation was terminated by cooling, and the reaction mixture was extracted with $2 \mathrm{ml}$ of ice-chilled chloroform. The aqueous layer was removed and mixed with $100 \mathrm{mg}$ of activated charcoal. The mixture was vortexed well and centrifuged at $1,500 \mathrm{x} \mathrm{g}$ for $10 \mathrm{~min}$ at $4^{\circ} \mathrm{C}$ to remove the charcoal. An aliquot $(0.5 \mathrm{ml})$ of the supernatant was added to $8 \mathrm{ml}$ of Scintisol fluid (Dojin Chemical Co., Kumamoto, Japan) in scintillation vials, vortexed, and measured for radioactivity. Blank values were subtracted, and the aromatase activity was expressed as fmol 
${ }^{3} \mathrm{H}_{2} \mathrm{O}$ released $/ \mathrm{min} / \mathrm{mg}$ protein. Protein concentration of the supernatant of the cell homogenate was determined by BCA Protein Assay Kit (Takara Bio Inc., Otsu, Japan).

Human breast cancer xenograft models. The premenopausal breast cancer xenograft model was established as follows. A suspension of MCF-7 cells ( $5 \times 10^{6}$ cells/mouse) was subcutaneously inoculated into the right flank of the BALB/c-nu/nu mice which had been subcutaneously implanted with slow-release estrogen pellets $(0.25 \mathrm{mg} /$ pellet $17 \beta$-estradiol; Innovative Research of America, Sarasota, FL) the day before tumor cell inoculation. Several weeks after tumor inoculation, mice bearing a tumor of $200-400 \mathrm{~mm}^{3}$ in volume were selected and were randomly allocated to control and treatment groups. Each group consisted of 8 mice. The mice received 6-week oral therapy with capecitabine (days 1-14, every 3 weeks) at maximum tolerated dose (MTD; $539 \mathrm{mg} / \mathrm{kg} /$ day) or two thirds MTD (359 mg/kg/day) and/or tamoxifen daily for 6 weeks at doses of 30 or $100 \mathrm{mg} / \mathrm{kg} /$ day.

The postmenopausal breast cancer xenograft model was established as follows. BALB/c-nu/nu mice were ovariectomized and subcutaneously implanted with slow-release androstenedione pellets $(1.5 \mathrm{mg} /$ pellet; Innovative Research of America) the day before tumor cell inoculation. MCF-7A25F3 cells $\left(5 \times 10^{6}\right.$ cells/mouse) were subcutaneously inoculated into the right flank of the mice. Mice bearing a tumor of $200-400 \mathrm{~mm}^{3}$ in volume were selected and were randomly allocated to control and treatment groups of 5 mice each. The mice received 6-week oral administration of capecitabine (days 1-14, every 3 weeks) at two thirds MTD and/or letrozole, at $0.1 \mathrm{mg} / \mathrm{kg} / \mathrm{day}$. In each model, control mice received vehicle alone.

Tumor volumes $(\mathrm{V})$ were estimated from the equation $\mathrm{V}=$ $a b^{2} / 2$, where $a$ and $b$ are the length and width of the tumor, respectively. Tumor volumes and body weights were monitored two or three times a week starting from the first day of the treatment.

Measurement of thymidine phosphorylase (TP). The tumor tissues were homogenized with a glass homogenizer in $10 \mathrm{mmol} / \mathrm{l}$ Tris buffer ( $\mathrm{pH}$ 7.4) containing $15 \mathrm{mmol} / \mathrm{l} \mathrm{NaCl}$, $1.5 \mathrm{mmol} / 1 \mathrm{MgCl}_{2}$ and $50 \mu \mathrm{mol} / 1$ potassium phosphate. The homogenates were then centrifuged at $10,000 \mathrm{xg}$ for $20 \mathrm{~min}$ at $4^{\circ} \mathrm{C}$, and the supernatants were stored at $-80^{\circ} \mathrm{C}$ until use. The TP level was measured by ELISA using monoclonal antibodies specific to human TP as described previously by Nishida et al (18). One unit corresponds to the TP level of the standard enzyme solution (extracts of human colon cancer HCT116 xenograft) which converts 5'-DFUR to 5-FU at a rate of $1 \mu \mathrm{g} \mathrm{5-FU/h} \mathrm{(18).}$ The protein concentration was determined using DC protein Assay Kit (Bio-Rad Laboratories, Hercules, CA).

Preparation of estrogen-responsive element-green fluorescence protein transfected cell line, MCF-7E10. The procedure for preparation of estrogen-responsive element-green fluorescence protein (ERE-GFP)-transfected cell line was described previously (16). Briefly, MCF-7 cells were transfected with the d2E-green fluorescent protein (GFP) vector alone (Clontech Lab., Inc., Palo Alto, CA) or carrying the ptk-estrogen-responsive element (ERE) insert using Trans IT LT-1 reagent (Takara Shuzo Co., Ltd., Tokyo, Japan) according to the manufacturer's instructions. After $24 \mathrm{~h}$, the cells were subjected to selection in growth medium containing geneticin $(1 \mathrm{mg} / \mathrm{ml})$. The ERE-GFP-MCF-7 clone that expressed high levels of GFP in the presence of estrogen, but not in the absence of estrogen, was selected (MCF-7E10).

Isolation of primary stromal cells from tumors and preparation of breast cancer extract. To obtain breast cancer extract, tissue specimens obtained from three premenopausal patients were minced in phenol red-free RPMI-1640 medium (PRF-RPMI; Sigma-Aldrich Co.) and suspended. The suspension was centrifuged $\left(600 \mathrm{x} \mathrm{g}, 10 \mathrm{~min}, 4^{\circ} \mathrm{C}\right)$ and the supernatant was further subjected to centrifugation $\left(12,000 \times \mathrm{g}, 10 \mathrm{~min}, 4^{\circ} \mathrm{C}\right)$ to obtain breast cancer extract. The isolation procedure of cancer stromal cells is similar to that described by Ackerman et al (19). The breast cancer tissue specimen obtained from a postmenopausal patient was minced in PRF-RPMI and suspended. The suspension was centrifuged $\left(600 \mathrm{x} \mathrm{g}, 10 \mathrm{~min}, 4^{\circ} \mathrm{C}\right)$ and the pellet was washed with Hanks' balanced salt solution (HBSS: Sigma-Aldrich Co.) and treated with the mixture of collagenase (1 mg/ml: Nitta Gelatin Co. Ltd., Osaka Japan), bovine serum albumin (40 mg/ml: Sigma-Aldrich Co.), glucose ( $2 \mathrm{mg}$ / ml: Wako Pure Chemical Industries, Osaka, Japan), antibioticantimycotic (Invitrogen, Corp.) and gentamicin $(50 \mu \mathrm{g} / \mathrm{ml}$ : Schering-Plough, NJ) for 2-3 $\mathrm{h}$ at $37^{\circ} \mathrm{C}$ followed by filtration through nylon mesh. The cells, including stromal cells, were recovered by centrifugation $\left(600 \times \mathrm{g}, 10 \mathrm{~min}, 4^{\circ} \mathrm{C}\right)$ and washed several times with HBSS. The cells were suspended in MEM- $\alpha$ medium (Invitrogen, Corp.) containing 10\% FBS (Tissue Culture Biologicals, Turale, CA) and cultured at $37^{\circ} \mathrm{C}$ in a humidified atmosphere of $5 \% \mathrm{CO}_{2}$ in air.

The study was approved by the Saitama Cancer Center (Saitama, Japan) Ethics Committee. Human breast cancer tissues were obtained from surgical specimens at the Saitama Cancer Center Hospital after obtaining informed consent from the patients.

Breast cancer extract ERE reporter gene assay and coculture ERE reporter gene assay. The breast cancer extract ERE reporter gene assays were carried out according to the method of Yamaguchi et al (20). The MCF-7E10 cells were cultured with $5 \%(\mathrm{v} / \mathrm{v})$ breast cancer tissue extract for 4 days in the presence of 5'-DFUR and/or 4-OHT. The cells were collected by mild trypsinization, and the number of cells expressing GFP was counted under fluorescence microscopy (16).

Coculture ERE reporter gene assays were performed as previously described (16). Briefly, MCF-7E10 cells and the stromal cells were precultured in PRF-RPMI containing $10 \%$ dextran-coated, charcoal-treated FBS (DCC-FBS; Hyclone Lab., UT) for $72 \mathrm{~h}$. Harvested stromal cells were seeded at $5 \times 10^{4} / \mathrm{ml}$ in a 24 -well multidish, and the next day, $5 \times 10^{4}$ MCF-7E10 cells were seeded on top of the adipose stromal cells with testosterone at $10^{-7} \mathrm{~mol} / \mathrm{l}$ as a substrate for aromatase. After being cocultured for 4 days in the presence of 5'-DFUR, an intermediate of capecitabine, and/or letrozole, the cells were collected by mild trypsinization, and the number of cells expressing GFP was counted under fluorescence microscopy. Data are expressed as a percentage of cells expressing GFP. The isobole method was performed in each experiment to analyze the mode of interaction between capecitabine and 


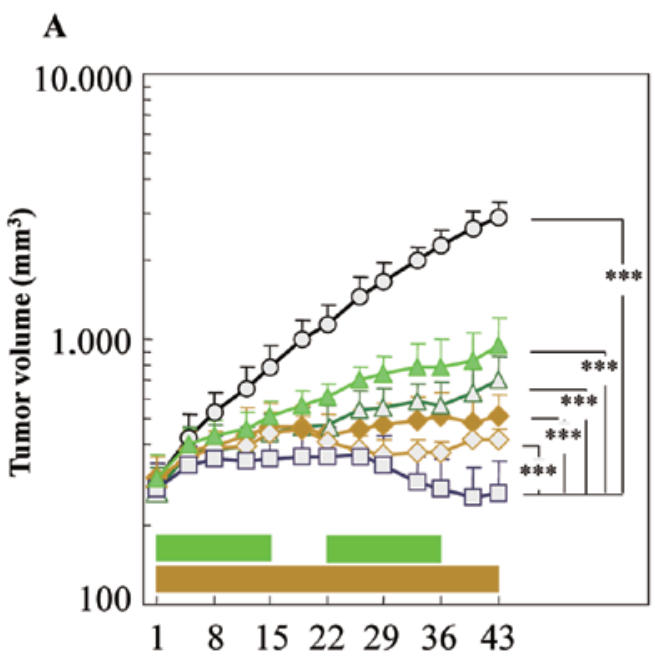

Days after treatment started

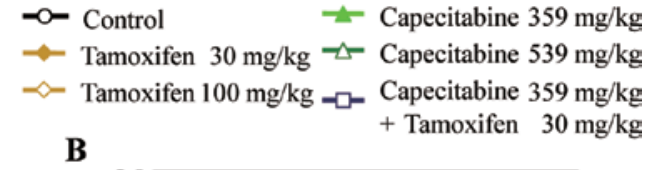

B

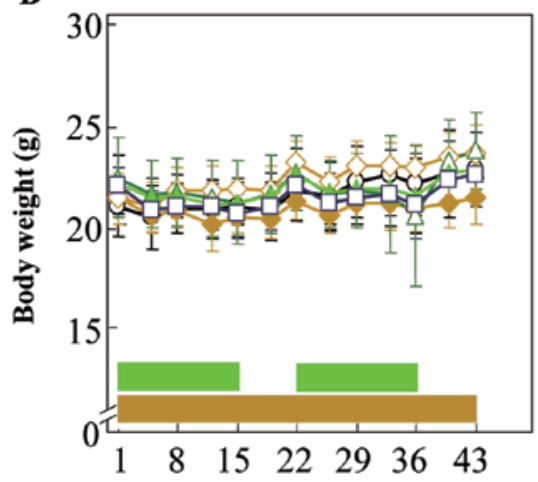

Days after treatment started

Figure 1. Antitumor activity of combination therapy of capecitabine with tamoxifen in a premenopausal breast cancer xenograft model. Mice were randomized into 6 groups of 8 mice each. Capecitabine ( 359 or $539 \mathrm{mg} / \mathrm{kg}$ ) was orally administered once a day from day 1 to day 14 and day 22 to day 35 (green horizontal bars). Tamoxifen (30 or $100 \mathrm{mg} / \mathrm{kg}$ ) was orally administered once a day for 6 weeks (brown horizontal bar). Data points indicate mean \pm SD of tumor volume (A) and mean \pm SD of body weight (B). Statistically significant differences versus capecitabine $359 \mathrm{mg} / \mathrm{kg}+\operatorname{tamoxifen~} 30 \mathrm{mg} / \mathrm{kg}$ group are shown with asterisks: ${ }^{* * *} \mathrm{p}<0.001$ by Wilcoxon test.

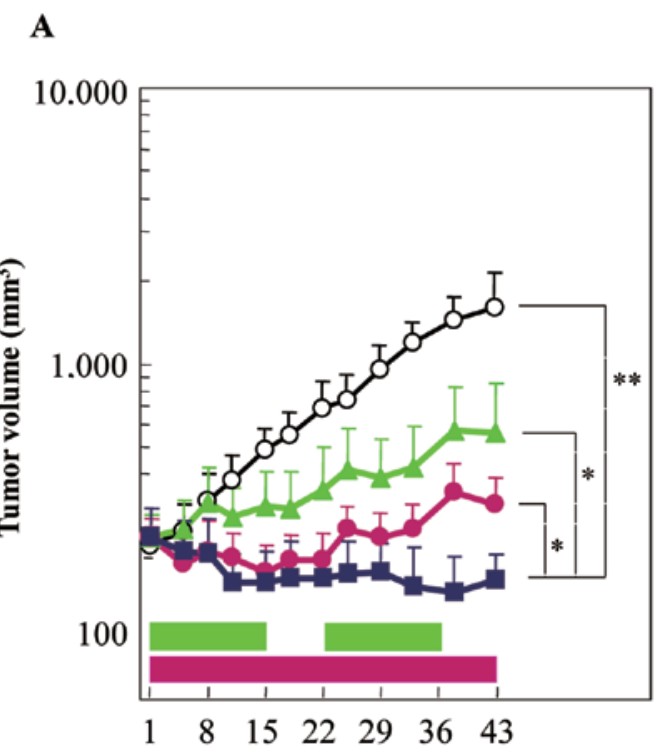

Days after treatment started

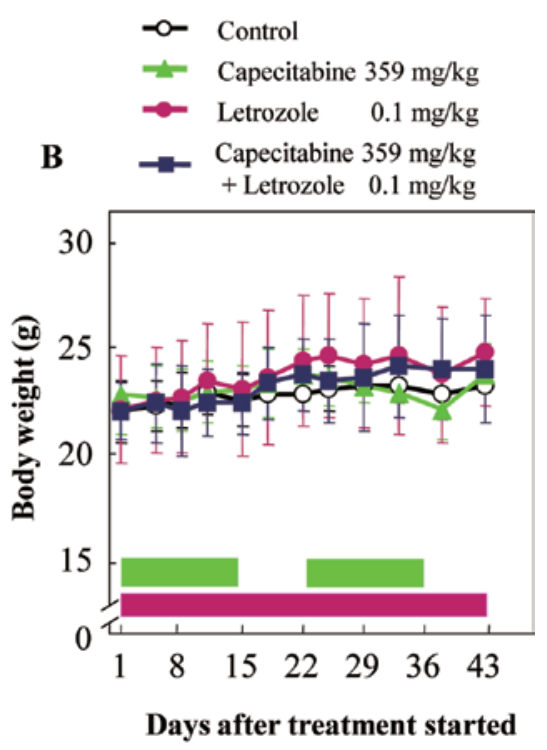

Figure 2. Antitumor activity of combination therapy of capecitabine with letrozole in a postmenopausal breast cancer xenograft model. Mice were randomized into 4 groups of 5 mice each. Capecitabine $(359 \mathrm{mg} / \mathrm{kg}$ ) was orally administered once a day from day 1 to day 14 and day 22 to day 35 (green horizontal bars). Letrozole $(0.1 \mathrm{mg} / \mathrm{kg}$ ) was orally administered once a day for 6 weeks (purple horizontal bar). Data points indicate mean $\pm \mathrm{SD}$ of tumor volume (A) and mean $\pm \mathrm{SD}$ of body weight (B). Statistically significant differences versus capecitabine $359 \mathrm{mg} / \mathrm{kg}+$ letrozole $0.1 \mathrm{mg} / \mathrm{kg}$ group are shown with asterisks: ${ }^{*} \mathrm{p}<0.05$, ${ }^{* *} \mathrm{p}<0.01$ by Wilcoxon test.

4-OHT/letrozole according to the method of Berenbaum MC (21).

Statistical analysis. The Wilcoxon test was used to detect the statistical differences in tumor volume, body weight and TP level. Statistical analyses were carried out using the SAS preclinical package (version 5.0; SAS Institute Inc., Tokyo, Japan). Differences were considered to be significant at $\mathrm{p}<0.05$.

\section{Results}

Establishment of MCF-7 aromatase-transfectant clone. Three aromatase-transfectant clones were selected by in vitro screening. The 3 clones were inoculated into ovariectomized BALB/c-nu/ nu mice which had been implanted with slow-release estradiol or androstenedione pellets to determine the tumor growth in response to each hormone in vivo. The clone, MCF-7A25F3, 
$\mathbf{A}$

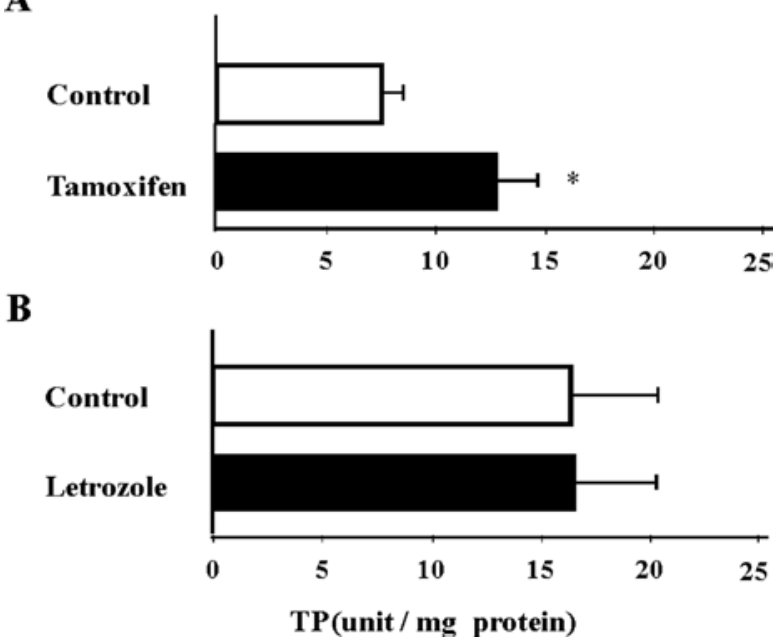

Figure 3. TP activity in tumor tissue after tamoxifen or letrozole treatment. Mice inoculated with MCF-7 or MCF-7A25F3 cells were randomly assorted to groups of 4 or 5 mice each. Tamoxifen (A) or letrozole (B) was orally administered once a day for 3 weeks and tumor tissues were excised on day 22. TP levels in tumor tissues were measured by ELISA. Data bars indicate mean \pm SD. Statistically significant differences versus control group are shown with asterisks: ${ }^{\mathrm{p}}<0.05$ by Wilcoxon test.

which showed the highest tumor growth was selected (data not shown). The MCF-7A25F3 cells were maintained in the same medium as MCF-7 cells supplemented with 10\% DCC-FBS and $350 \mu \mathrm{g} / \mathrm{ml}$ geneticin. The tumor of parent MCF-7 cells grew in BALB/c-nu/nu mice with estrogen pellet but not in BALB/c $\mathrm{nu} / \mathrm{nu}$ mice with androstenedione pellet (data not shown). The aromatase activity in MCF-7A25F3 cell homogenate was 420 -fold higher ( $50.4 \mathrm{fmol} / \mathrm{min} / \mathrm{mg}$ protein) than that of parent MCF-7 ( $0.12 \mathrm{fmol} /$ minute/mg protein) measured by the ${ }^{3} \mathrm{H}_{2} \mathrm{O}$ release from $1 \beta-{ }^{3} \mathrm{H}$-androstenedione. To confirm the androstenedione-dependent growth of MCF-7A25F3 cells, tumor growth inhibition by letrozole was examined in vivo. MCF-7A25F3 cells were inoculated into BALB/c nu/nu mice which had been ovariectomized and subcutaneously implanted with androstenedione pellets. BALB/c nu/nu mice received daily oral administration of $0.03-3 \mathrm{mg} / \mathrm{kg}$ of letrozole for 6 weeks. Letrozole significantly inhibited the tumor growth in a dose-dependent manner with the maximum effect at $1-3 \mathrm{mg} / \mathrm{kg}$ (data not shown).

Antitumor activity of capecitabine in combination with tamoxifen in a premenopausal xenograft model. The antitumor activity of capecitabine in combination with tamoxifen was evaluated using MCF-7 premenopausal breast cancer xenograft model. The dosages of these agents were determined by a preliminary dose-finding experiment (data not shown). To detect an interaction between the two agents distinctly, suboptimal doses of each drug were used in combination therapy. The MTD for capecitabine administered daily for 2 weeks in 3-week cycles in this model was determined as $539 \mathrm{mg} / \mathrm{kg} /$ day in a previous experiment $(22,23)$. The daily oral administration of tamoxifen for 6 weeks showed maximum antitumor activity at a dose of $100 \mathrm{mg} / \mathrm{kg}$. Therefore, in the present combination therapy experiments, $359 \mathrm{mg} / \mathrm{kg}$ (2/3 MTD) for capecitabine, and the dose of $30 \mathrm{mg} / \mathrm{kg}$ (1/3 of maximum effective dose) for tamoxifen were selected.
As shown in Fig. 1A, both capecitabine and tamoxifen showed only moderate antitumor activities at selected doses for each agent. In contrast, the antitumor activity of combination therapy was significantly superior to the antitumor activities of monotherapy of each agent $(\mathrm{p}<0.001)$. In addition, the combination therapy produced statistically significant inhibition of tumor growth compared with tamoxifen at $100 \mathrm{mg} / \mathrm{kg}$ $(\mathrm{p}<0.001)$ or capecitabine at $539 \mathrm{mg} / \mathrm{kg}$ in monotherapy. None of the treatment groups in this experiment showed significant weight loss during the treatments (Fig. 1B).

Antitumor activity of capecitabine in combination with letrozole in a postmenopausal xenograft model. The anti-tumor activity of capecitabine in combination with letrozole was evaluated in the postmenopausal breast cancer xenograft model using MCF-7A25F3 cells. To detect the interaction between the two agents distinctly, the dose of $0.1 \mathrm{mg} / \mathrm{kg}$ for letrozole, which was $1 / 30$ of the maximum effective dose (data not shown) and the dose of $359 \mathrm{mg} / \mathrm{kg}(2 / 3 \mathrm{MTD})$ for capecitabine, were used for combination therapy as a suboptimal dose in each monotherapy. The combination therapy of capecitabine with letrozole showed significantly higher antitumor activity than each monotherapy (Fig. 2A). None of the treatment groups in this experiment showed significant weight loss during the treatments (Fig. 2B).

TP levels in the tumor tissues. Mice inoculated with MCF-7 or MCF-7A25F3 cells were randomly assorted into groups of 4 and 5 mice each, respectively. The TP levels in tumor tissues were measured 22 days after treatment started. The TP level of MCF-7 tumor was significantly increased $(12.8 \pm 2.0 \mathrm{U} / \mathrm{mg}$ protein) by tamoxifen $(30 \mathrm{mg} / \mathrm{kg} /$ day) treatment for 3 weeks compared with vehicle-treated group $(7.6 \pm 1.1 \mathrm{U} / \mathrm{mg}$ protein) (Fig. 3A). On the other hand, the TP level of MCF-7A25F3 tumor was higher $(16.4 \pm 3.8 \mathrm{U} / \mathrm{mg}$ protein) than that of MCF-7 parent tumor and no further upregulation of TP was observed by $0.1 \mathrm{mg} / \mathrm{kg}$ of letrozole treatment $(16.6 \pm 3.8 \mathrm{U} / \mathrm{mg}$ protein) (Fig. 3B).

Breast cancer extract ERE reporter gene assay and coculture ERE reporter gene assay. The inhibitory effect of 5'-DFUR in combination with 4-OHT, an active form of tamoxifen, on estrogen-responding cells was evaluated using breast cancer extract ERE reporter gene assay (Fig. 4A). The percentage of GFP-positive cells was decreased by the addition of 5'-DFUR or 4-OHT in a concentration-dependent manner. The analysis by isobole method showed a synergistic effect of 5'-DFUR and 4-OHT (Fig. 4B).

The inhibitory effect of 5'-DFUR in combination with letrozole on estrogen-responding cells was evaluated using coculture ERE reporter gene assay (Fig. 4C). The percentage of GFP-positive cells was decreased by the addition of 5'-DFUR or letrozole in a concentration-dependent manner. The isobole analysis showed an additive effect of 5'-DFUR and letrozole (Fig. 4D).

\section{Discussion}

The combination effects of antiestrogens and chemotherapeutic agents have been controversial for many years. Specifically, 
A

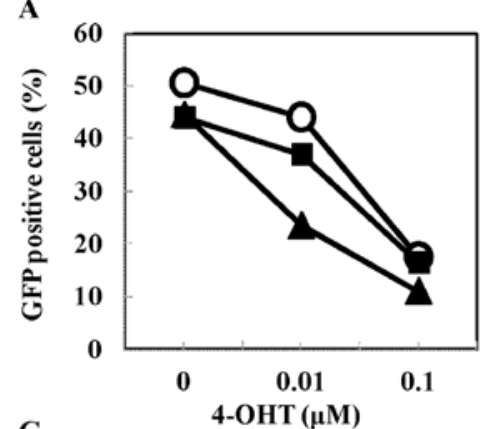

,

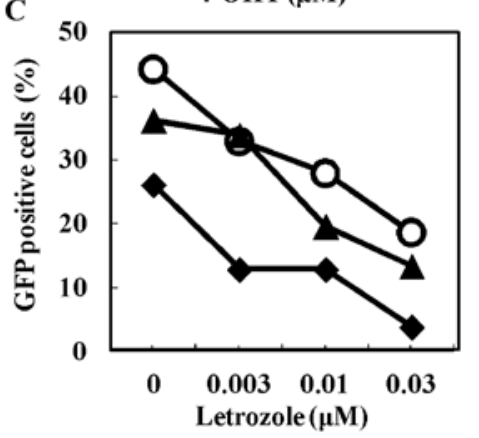

B
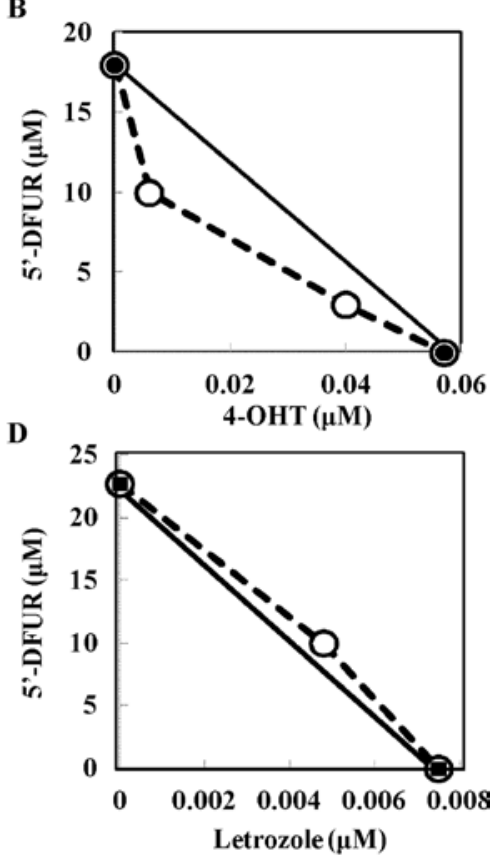

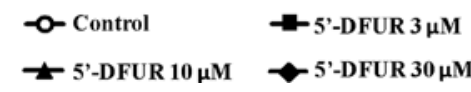

Figure 4. Combination effect of 5'-DFUR with 4-OHT, or 5'-DFUR with letrozole in ERE reporter gene assay. The MCF-7E10 cells were cultured with various concentrations of 5'-DFUR and 4-OHT in the presence of breast cancer extract for 4 days, and the number of cells expressing GFP was counted. Data were expressed as a percentage of GFP-expressing cells against the observed number of MCF-7E10 cells (A). Isobolograms of the interactions between 5'-DFUR and 4-OHT were taken at the point of 30\% GFP-positive cells (B). The MCF-7E10 cells were cultured with various concentrations of 5'-DFUR and letrozole in the presence of breast cancer stromal cells for 4 days, and the number of cells expressing GFP was counted. Data were expressed as a percentage of GFPexpressing cells against the observed number of MCF-7E10 cells (C). Isobolograms of the interactions between 5'-DFUR and letrozole were taken at the point of 30\% GFP-positive cells (D).

chemoendocrine combinations of tamoxifen with chemotherapy containing 5-FU revealed both positive and negative results. Fisher et al reported the prolongation of the disease-free interval in the therapy of tamoxifen with L-phenylalanine mustard and 5-FU in patients with primary operable breast cancer (4). The IBCSG Trial VII reported that early CMF added to tamoxifen significantly improved 5-year disease-free survival in postmenopausal breast cancer patients (5). On the other hand, Rivkin et al reported that combination treatment of CMFVP (cyclophosphamide, methotrexate, fluorouracil, vincristine, predonisone) chemotherapy with tamoxifen has not been shown to be superior to tamoxifen alone in the treatment of postmenopausal women with node-positive, ER-positive, operable breast cancer (2). Pritchard et al reported that there were no significant differences in overall survival, recurrence-free survival, locoregional recurrence-free survival and distant recurrence-free survival between the tamoxifen treatment group and tamoxifen plus CMF group in postmenopausal breast cancer patients (3). Perry et al reported that no difference was evident among postmenopausal patients in overall response rate and duration of responses between CAF (cyclophosphamide, adriamycin, 5-fluorouracil) and CAF plus tamoxifen, and that no difference of response rates was shown among premenopausal patients between CAF and CAF plus tamoxifen (24). However, in in vitro, a study on mammary carcinoma cell lines showed that the cytotoxic effects of cotreatment of 5'-DFUR with tamoxifen were dependent on tamoxifen concentration and treatment duration (25). Since 5 -DFUR is known to be converted to 5-FU by TP and it was also reported that tamoxifen upregulated TP in breast cancer cells (26), data suggest that there is a mechanism for a potentially favorable outcome of chemoendocrine therapy with tamoxifen and such chemotherapeutic agents as were activated by TP.

In this study, we showed the antitumor efficacy of chemoendocrine therapy using capecitabine as a chemotherapeutic agent, in premenopausal as well as postmenopausal breast cancer xenograft models. Capecitabine has been approved for breast cancer in many countries due to proven efficacy and tolerability in patients with metastatic breast cancer $(27,28)$. Tamoxifen has been the most widely used anti-hormonal agent for many years in the first line treatment of ER-positive breast cancers, and has a profound impact on breast cancer mortality $(8,9,29)$. Letrozole has been used for the breast cancers of postmenopausal women, and Mouridsen et al demonstrated the superiority of letrozole to tamoxifen in first-line endocrine therapy in postmenopausal breast cancer patients (30).

In the present study, we examined the antitumor efficacy of combination therapy of capecitabine with tamoxifen or with letrozole in premenopausal and postmenopausal status, respectively. For a premenopausal model, ER-positive MCF-7 human breast cancer cells were used. For a postmenopausal model, we prepared the MCF-7 aromatase transfectant clone, MCF-7A25F3. The feature of MCF-7A25F3 cells as aromatase 
transfectants was confirmed by the estradiol- and androstenedione-dependent growth in vivo and the increased (420-fold) aromatase activity in MCF-7A25F3 cells compared with parent MCF-7 cells.

Using the established premenopausal and postmenopausal human breast cancer xenograft models, we evaluated the antitumor efficacy of combination therapy of capecitabine with tamoxifen or letrozole. In each model, dose and schedule of capecitabine was determined according to the study of Sawada et al $(22,23)$. To make the combination effect clear, MTD or sub-MTD of capecitabine and suboptimal dose of tamoxifen or letrozole were used according to the preliminary experiments (data not shown). In both models, combination therapy clearly showed the supra-additive antitumor activity to either monotherapy. In the premenopausal model, the combination therapy of capecitabine at $359 \mathrm{mg} / \mathrm{kg}$ (2/3 MTD) with tamoxifen at $30 \mathrm{mg} / \mathrm{kg}$ showed a significantly higher antitumor activity than each monotherapy (Fig. 1A). The combination therapy showed higher antitumor activity than MTD (539 mg/ $\mathrm{kg}$ ) of capecitabine or $100 \mathrm{mg} / \mathrm{kg}$ of tamoxifen. In the postmenopausal model, the combination therapy of capecitabine at $2 / 3 \mathrm{MTD}$ with letrozole at $0.1 \mathrm{mg} / \mathrm{kg}$ showed significantly higher antitumor activity than either monotherapy (Fig. 2A). Concerning the therapeutic experiment of breast cancer aromatase transfectant with letrozole, Long et al reported that suppression of tumor growth was observed for at least 44 weeks in MCF-7Ca aromatase transfectant xenograft model in which letrozole was subcutaneously injected daily (10 $\mu \mathrm{g} /$ day $)$ (31). However, we showed the antitumor effect of letrozole by oral administration, which is consistent with the clinical administration route. No augmentation of toxicity in terms of body weight loss was observed in the combination therapy in either model (Figs. 1B and 2B). The results indicate that both chemoendocrine therapy regimens may be applicable to breast cancer patients.

Capecitabine generates the active substance 5-FU selectively in tumors by using three enzymes located in the liver and in tumors $(22,23,32)$. The final step is the conversion of the intermediate metabolite 5'-DFUR to 5-FU by TP in tumors and this conversion appeared to be a rate-limiting step for the efficacy of capecitabine. Ishikawa et al reported that the efficacy of capecitabine correlated with TP activity in tumor tissues using human cancer xenograft models (6). TP is upregulated in tumors by receiving stress such as hypoxia, radiation and chemotherapeutic damage. Several types of cytokines such as interleukin-1, tumor necrosis factor- $\alpha$, interferon- $\gamma$ also upregulate the expression of TP in malignant cells (33). Endo et al have previously reported that several types of antitumor drugs, such as taxanes, cyclophosphamide and oxaliplatin, upregulated TP in tumor tissues and that capecitabine in combination with these TP upregulators showed synergistic antitumor activity in xenograft models (32). Ustunomiya et al reported the upregulation of TP in T47D breast cancer cells treated with tamoxifen in vitro (26) and also Evans et al reported the induction of hypoxia by tamoxifen in MCF-7 xenografts (34). These findings prompted us to examine the TP activity in the tumor tissue of mice treated with tamoxifen and letrozole. Consequently, we found that the TP activity in MCF-7 tumor tissue of mice treated with tamoxifen was significantly increased compared to that of control mice in the premenopausal model (Fig. 3A). Thus, the increased efficacy of combination therapy of capecitabine with tamoxifen may, at least partly, be explained by the upregulation of TP by tamoxifen in tumor tissue facilitating the conversion of capecitabine to 5-FU. On the other hand, in the postmenopausal model, the increased efficacy of combination therapy of capecitabine with letrozole could not be explained by TP upregulation because no TP increase was observed by letrozole treatment, suggesting that mechanisms other than TP upregulation are involved in the efficacy of this combination therapy (Fig. 3B).

Next, we tried to analyze the effect of the combinations capecitabine with tamoxifen or capecitabine with letrozole in vitro. For this purpose, we established ERE reporter gene assay, to mimic premenopausal or postmenopausal breast cancer $(16,20)$. For the combination effect of capecitabine with tamoxifen, MCF-7E10 cells were cultured with breast cancer extract in the presence of various concentrations of 5'-DFUR and 4-OHT, an active metabolite of tamoxifen (35). To make the assay system closer to the clinical conditions of premenopausal breast cancer, human breast cancer extracts were used as a source of estrogen and other unknown factors which may affect the ER signals and cell proliferation. Both 5'-DFUR and 4-OHT decreased the percentage of GFP-positive cells in a dose-dependent manner. The analysis by isobole method showed the mode of the combination effect of the two drugs to be synergistic. The mechanism of the synergy was considered to come from the TP upregulation in MCF-7E10 cells by tamoxifen facilitating the generation of 5-FU from 5'-DFUR. The combination effect of capecitabine with letrozole was examined using coculture ERE reporter gene assay which corresponds to postmenopausal breast cancer. In this assay, exogenously added testosterone was converted into estrogen by aromatase in stromal cells isolated from breast cancer tissues. Both 5'-DFUR and letrozole decreased the percentage of GFP-positive cells in a dose-dependent manner. The analysis by isobole method showed the mode of the combination effect of the two drugs to be additive. The results indicated that 5'-DFUR and letrozole work independently and do not interfere with each other. Unexpectedly, the results of the in vitro letrozole experiment did not coincide with the in vivo results. The reason for this discrepancy could not be delineated in the present study.

In conclusion, several types of therapeutic agents including chemotherapeutic agents, endocrine therapy agents and molecular targeted medicines are now available for the treatment of breast cancer. To conduct clinical studies, research demonstrating the scientific rationale is required. In the present study, we showed the efficacy of combination therapy of capecitabine with tamoxifen or letrozole for a premenopausal and postmenopausal breast cancer model, respectively. The usefulness of these combinations in patients needs to be confirmed in clinical trials.

\section{References}

1. Hug V, Hortobagyi GN, Drewinko B and Finders MJ: Tamoxifencitrate counteracts the antitumor effects of cytotoxic drugs in vitro. Clin Oncol 3: 1672-1677, 1985.

2. Rivkin SE, Green S, Metch B, Cruz AB, Abeloff MD, Jewell WR, Costanzi JJ, Farrar WB, Minton JP and Osborne CK: Adjuvant CMFVP versus tamoxifen versus concurrent CMFVP and tamoxifen for postmenopausal, node-positive, and estrogen receptor-positive breast cancer patients: a southwest oncology group study. J Clin Oncol 12: 2078-2085, 1994. 
3. Pritchard KI, Paterson AHG, Fine S, et al: Randomized trial of cyclophosphamide, methotrexate, and fluorouracil chemotherapy added to tamoxifen as adjuvant therapy in postmenopausal women with node-positive estrogen and/or progesterone receptor positive breast cancer: a report of the national cancer institute of Canada clinical trials group. J Clin Oncol 15: 2302-2311, 1997.

4. Fisher B, Redmond C, Brown A, et al: Adjuvant chemotherapy with and without tamoxifen in the treatment of primary breast cancer: 5-year results from the national surgical adjuvant breast and bowel project trial. J Clin Oncol 4: 459-471, 1986.

5. International Breast Cancer Study Group: Effectiveness of adjuvant chemotherapy in combination with tamoxifen for nodepositive postmenopausal breast cancer patients. J Clin Oncol 15 : 1385-1394, 1997.

6. Ishikawa T, Sekiguchi F, Fukase Y, Sawada N and Ishitsuka H: Positive correlation between the efficacy of capecitabine and doxifluridine and the ratio of thymidine phosphorylase to dihydropyrimidine dehydrogenase activities in tumors in human cancer xenografts. Cancer Res 58: 685-690, 1998.

7. Harvey JM, Clark GM, Osborne CK and Allred DC: Estrogen receptor status by immunohistochemistry is superior to the ligand-binding assay for predicting response to adjuvant endocrine therapy in breast cancer. J Clin Oncol 117: 1474-1481, 1999.

8. Fisher B, Costantino JP, Wickerham DL, et al: Tamoxifen for the prevention of breast cancer: Current status of the national surgical adjuvant breast and bowel project P-1 study. J Natl Cancer Inst 97: 1652-1662, 2005.

9. Alkner S, Bendahl P-O, Ferno M, Nordenskjold B, Ryden L, South Swedish and South-East Swedish Breast Cancer Groups: Tamoxifen reduces the risk of contralateral breast cancer in premenopausal women: Results from a controlled randomized trial. Eur J Cancer 45: 2496-2502, 2009.

10. Pasqualini JR, Chetrite J and Blacker C: Concentration of estrone, estradiol, and estrone sulfate and evaluation of sulfatase and aromatase activities in pre- and postmenopausal breast cancer patients. J Clin Endocrinol Metab 81: 460-464, 1996.

11. Miki Y, Suzuki T, Tazawa C, et al: Aromatase localization in human breast cancer tissues: Possible interactions between intratumoral stromal and parenchymal cells. Cancer Res 67: 3945-3954, 2007.

12. O'Neill JS and Miller WR: Aromatase activity in breast adipose tissue from women with benign and malignant breast disease. $\mathrm{Br}$ J Cancer 56: 601-604, 1987.

13. Coates AS, Keshavish A and Thurliman B: Five years letrozole compared with tamoxifen as initial adjuvant therapy for postmenopausal women with endocrine-responsive early breast cancer: update of study BIG 1-98. J Clin Oncol 25: 486-492, 2007.

14. The Breast International Group (BIG) 1-98 collaborative group: A comparison of letrozole and tamoxifen in postmenopausal women with early breast cancer. N Engl J Med 29: 2747-2758, 2005

15. Yamaguchi Y and Hayashi S: Estrogen-related cancer microenvironment of breast carcinoma. Endocrine J 56: 1-7, 2009.

16. Yamaguchi Y, Takei H, Suemasu K, Kobayashi Y, Kurosumi M, Harada N and Hayashi S: Tumor-stromal interaction through the estrogen-signaling pathway in human breast cancer. Cancer Res 65: 4653-4662, 2005

17. Zhou D, Pompon D and Chen S: Stable expression of human aromatase complementary DNA in mammalian cells: Useful system for aromatase inhibitor screening. Cancer Res 50: 6949-6955, 1990.

18. Nishida M, Hino A, Mori K, Matsumoto T, Yoshikubo T and Ishitsuka H: Preparation of anti-human thymidine phosphorylase monoclonal antibody useful for detecting the enzyme levels in tumor tissues. Biol Pharma Bull 19: 1407-1411, 1996.
19. Ackerman GE, Smith ME, Mendelson CR, MacDonald PC and Simpson ER: Aromatization of androstendione by human adipose tissue stromal cells in monolayer culture. J Clin Endocrinol Metab 53: 412-417, 1981.

20. Yamaguchi Y, Takei H, Kurosumi M, Seino Y and Hayashi S: Regulation of growth and estrogen signals by tumor microenvironment in human breast cancers. Proceedings of the 12th International Congress on Hormonal Steroids and Hormones \& Cancer, 2006.

21. Beenbaum MC: What is synergy? Pharmacological Rev 41: 93-141, 1989.

22. Sawada N, Ishikawa T, Fukase Y, Nishida M, Yoshikubo T and Ishitsuka $\mathrm{H}$ : Induction of thymidine phosphorylase activity and enhancement of capecitabine efficacy by taxol/taxotere in human cancer xenograft. Clin Cancer Res 4: 1013-1019, 1998.

23. Sawada N, Kondoh K and Mori K: Enhancement of capecitabine efficacy by oxaliplatin in human colorectal and gastric cancer xenografts. Oncol Rep 18: 775-778, 2007.

24. Perry MC, Kardinal CG, Weinberg V, Ginsberg AJ, Hughes A and Wood W: Chemotherapy with cyclophosphamide, adriamycin, and 5-fluorouracil compared to chemotherapy plus hormonal therapy with tamoxifen in the treatment of advanced breast cancer: an interim analysis. J Steroid Biochem 23: 1135-1140, 1895.

25. Bollig A, Du QQ, Fan ST, Yu B, Sarkar FH and Liao J: Combination of 5-deoxy-5-fluorouridine and tamoxifen show cell-type specific antagonistic and cooperative effects on cytotoxicity in human mammary carcinoma cells. Oncol Rep 14: 177-183, 2005.

26. Utsunomiya $H$, Ueshima $H$, Kawashiro $M$, Yang Q, Nakamura $M$, Sakurai Y, Mori I and Kakudo K: Thymidine phosphorylase is regulated by tamoxifen in T47D breast cancer cell line. Breast Cancer 9: 107-110, 2002.

27. Erschler WB: Capecitabine monotherapy: Safety and effectiveness of treatment for metastatic breast cancer. Oncologist 11: 325-335, 2006

28. Venturini M,Paridaens R, Rossner D, Valamatzis MW, Nortier JWR, Salzberg M, Rodrigues H and Bell R: An open-label, multicenter study of outpatient capecitabine monotherapy in 631 patients with pretreated advanced breast cancer. Oncologist 72: 51-57, 2007.

29. Early Breast Cancer Trialists' Collaborative Group (EBCTCG): Effect of chemotherapy and hormonal therapy for early breast cancer on recurrence and 15-year survival: an overview of the randomized trials. Lancet 365: 1687-1717, 2005.

30. Mouridsen H, Gershanovich M, Sun Y, et al: Phase III study of letrozole versus tamoxifen as first-line therapy of advanced breast cancer in postmenopausal women: Analysis of survival and update of efficacy from the international letrozole breast cancer group. J Clin Oncol 21: 2101-2109, 2003.

31. Long BJ,Jelovac D, Handratta V, Thianotanawat A, MacPherson N, Ragaz J, Goloubeva OG and Brodie AM: Therapeutic strategies using the aromatase inhibitor letrozole and tamoxifen in breast cancer model. J Natl Cancer Inst 96: 456-465, 2004.

32. Endo M, Shinbori N, Fukase Y, Sawada N, Ishikawa T, Ishituka H and Tanaka Y: Induction of thymidine phosphorylase expression and enhancement of efficacy of capecitabine or 5'-deoxy-5-fluorouridine by cyclophosphamide in mammary tumor models. Int J Cancer 83: 127-134, 1999.

33. Toi M, Bando H, Horiguchi S, Takada M, Kataoka A, Ueno T, Saji S, Muta M, Funata N and Ohno S: Modulation of thymidine phosphorylase by neoadjuvant chemotherapy in primary breast cancer. Br J Cancer 90: 2338-2343, 2004.

34. Evans SM, Koch CJ, Laughlin KM, Jenkins WT, Winkle TV and Wilson DF: Tamoxifen induces hypoxia in MCF-7 xenografts. Cancer Res 57: 5155-5161, 1997.

35. Jordan VC: New insights into the metabolism of tamoxifen and its role in the treatment and prevention of breast cancer. Steroids 72: 829-842, 2007. 